\title{
A MICROFABRICATED GAS CHROMATOGRAPH FOR SUB-PPB DETERMINATIONS OF TCE IN VAPOR INTRUSION INVESTIGATIONS
}

\author{
Hungwei Chang, ${ }^{l}$ Sun Kyu Kim, ${ }^{l}$ Thitiporn Sukaew, ${ }^{1}$ Forest Bohrer, ${ }^{2}$ Edward T. Zellers ${ }^{1,2}$ \\ Center for Wireless Integrated Microsystems (WIMS), University of Michigan, Ann Arbor, MI, USA \\ Departments of ${ }^{1}$ Environmental Health Sciences and ${ }^{2}$ Chemistry, University of Michigan, Ann Arbor, MI, USA
}

\begin{abstract}
A microfabricated gas chromatograph $(\mu \mathrm{GC})$ is described and its application to the determination of low-parts-per-billion ( $\mathrm{ppb}$ ) concentrations of trichloroethylene (TCE) in mixtures is demonstrated, relevant to investigations of vapor intrusion (VI) into homes and offices. The system employs a MEMS focuser, dual MEMS separation columns, and MEMS interconnects along with a microsensor array. These are interfaced to a (non-MEMS) pre-trap and high-volume sampler module in order to reduce overall analysis time. The response patterns generated from the sensor array for each vapor are combined with the chromatographic retention time to identify and differentiate the components of VOC mixtures. All functions are controlled by a Labview routine written in house. A chemometric method based on multivariate curve resolution has also been developed for analyzing partially resolved mixture components. First results are presented of the capture, separation, recognition, and quantification of TCE in a mixture at $4 \mathrm{ppb}$, with a projected detection limit of 50 parts-per-trillion.
\end{abstract}

\section{INTRODUCTION}

The measurement of volatile organic compounds (VOC) in complex mixtures is critical to numerous public health efforts. Current VOC monitoring instrumentation is too large and expensive for routine implementation in many such applications. While stand-alone microsensor arrays (so-called electronic noses) can be used for the determination of individual VOCs in simple mixtures $[1,2]$, reliable quantitative analysis of complex gas/vapor mixtures with microsensor arrays demands chromatographic separation prior to measurement. Several reports have appeared over the past decade on microfabricated gas chromatographic $(\mu \mathrm{GC})$ separation columns coupled with one or more sampling, pretreatment and/or detection devices. However, reports on fully integrated $\mu \mathrm{GC}$ systems that incorporate microfabricated sampling/preconcentration, separation, and detection devices are relatively rare [3-6].

Vapor intrusion (VI) refers to VOC infiltration into buildings overlying contaminated soil or groundwater. VI is an emerging problem in the U. S., affecting thousands of civilian and military locations [7]. TCE is the most common contaminant of concern at VI-impacted sites, where concentrations in homes and buildings have been documented in the low-parts-per-billion to mid-partsper-trillion (ppt) range. In response to the need for inexpensive onsite monitoring instrumentation, we are developing a fieldable prototype $\mu \mathrm{GC}$ whose design and operating parameters are tailored for the determination of TCE from VI in near-real-time in the presence of common interfering VOCs. Performance specifications include a limit of detection (LOD) of < $100 \mathrm{ppt}$ of TCE, discrimination of TCE from typical indoor contaminants, and a total analytical cycle period of $<30$ minutes.

The schematic diagram of the $\mu \mathrm{GC}$, which has been named SPIRON, is shown in Figure 1 along with images of the core microfabricated components. This microsystem builds on previous work in our group on partially-integrated or singlecolumn prototypes $[3,4]$ and on component-level testing of critical

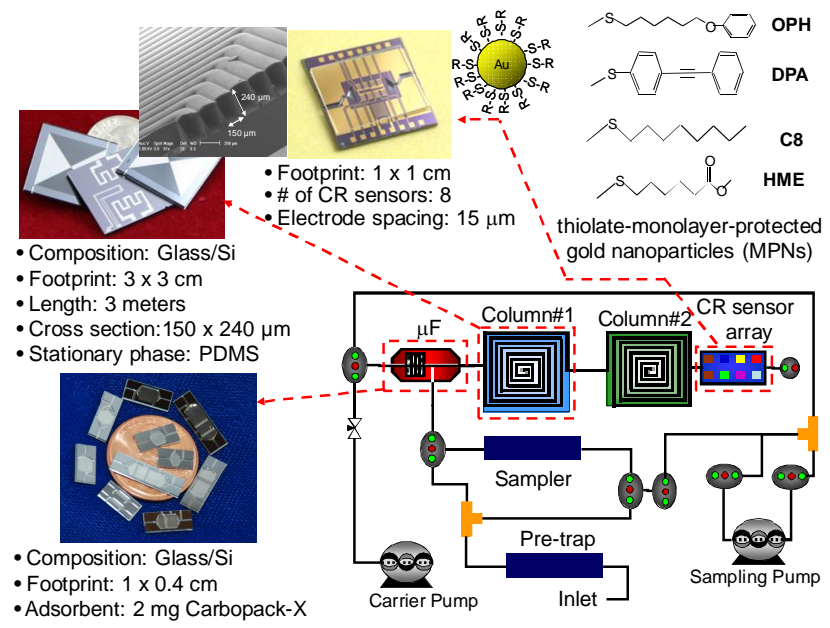

Figure 1: The SPIRON $\mu G C$ layout diagram and images of major components, microfocusers, dual 3-m microcolumns,, and nanoparticle-coated chemiresistor-array detector

microsystem elements [8-11]. The key components are an adsorbent-packed DRIE-Si/glass single-stage focuser $(\mu \mathrm{F})$, dual 3meter DRIE-Si/glass microcolumns and a chemiresistor (CR) sensor array incorporating thiolate-monolayer-protected gold nanoparticle (MPNs) films as sensing materials on interdigital electrodes [3]. To measure TCE in the desired concentration and analytical time ranges, a front-end high-volume sampler (nonMEMS) was added. In addition, a pre-trap (non-MEMS) was added to filter out low volatility interferences that might degrade system performance after prolonged use.

An analytical cycle comprises four steps. First, air samples are passed through the pre-trap and collected on the high-volume sampler at a flow rate of $\sim 1 \mathrm{~L} / \mathrm{min}$. Second, the VOCs captured on the sampler are thermally desorbed onto the $\mu \mathrm{F}$ at a low flow rate. Third, the $\mu \mathrm{F}$ is rapidly heated to inject the VOC mixture onto the first of two microcolumns for separation and detection of eluting peaks by the sensors in the CR array. Finally, the identification and quantification of each VOC is performed by combining retention time with array response patterns in chemometric analyses.

The work described herein focuses on various aspects of microsystem integration and performance. This includes the integration of the core MEMS components on a PCB with fluidic and electrical interconnections to supporting devices; the design, optimization, and integration of the front-end pre-trap, highvolume sampler, and $\mathrm{mF}$; demonstration of the analysis of TCE in a test mixture at low concentrations with the integrated microsystem, and methodology for chemometric discrimination of TCE from co-contaminants with similar elution times.

\section{RESULTS AND DISCUSSION \\ System Components and Integration}

The key components of the SPIRON $\mu \mathrm{GC}$ are presented in the prototype (mock-up) shown in Figure 2. The single-stage $\mu \mathrm{F}$ has 


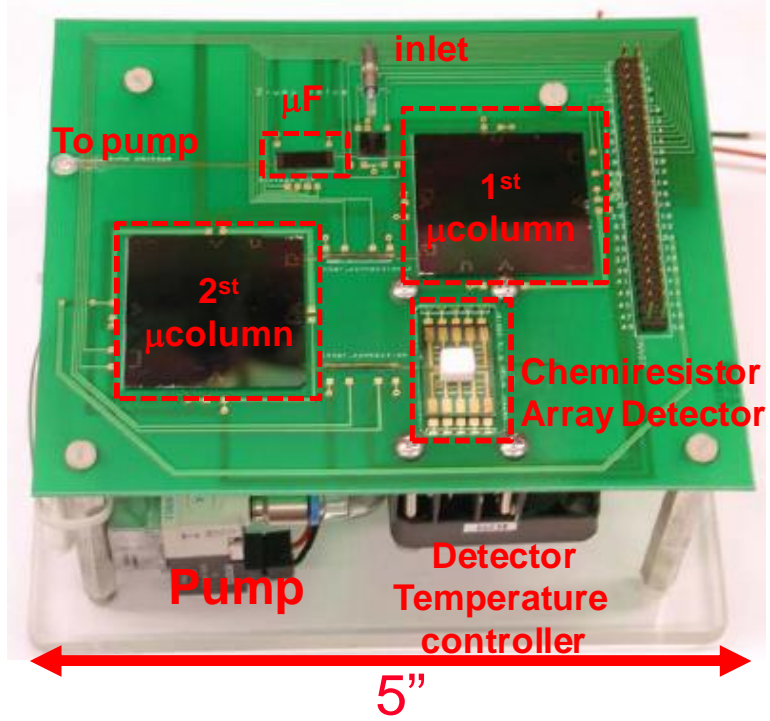

Figure 2: The SPIRON $\mu G C$ prototype (mock-up).

overall dimensions of $9.76 \mathrm{~mm} \times 4.18 \mathrm{~mm} \times 0.6 \mathrm{~mm}$ and was fabricated from double-polished $\mathrm{Si}$ wafers $(0.5 \mathrm{~mm}$ thick). Deepreactive-ion-etching (DRIE) was used to form a $3.2 \mathrm{~mm}$ x $3.45 \mathrm{~mm}$ cavity in the lower section of the device as well as to define inlet and outlet ports and an additional port for adsorbent filling ( 0.40 $\mathrm{mm})$. The device was capped with an anodically bonded Pyrex plate. A Ti/Pt RTD was evaporated onto the backside along with $\mathrm{Cr} / \mathrm{Au}$ contact pads for bulk heating of the device. A $2.3 \mathrm{mg}$ quantity of a graphitized carbon adsorbent (Carbopack X, specific surface area of $250 \mathrm{~m}^{2} / \mathrm{g}$ ) was sieved and then drawn into the $\mu \mathrm{F}$ by gentle suction and retained with DRIE pillars within the cavity blocking the exit port. Deactivated fused silica capillaries are used for fluidic interconnections.

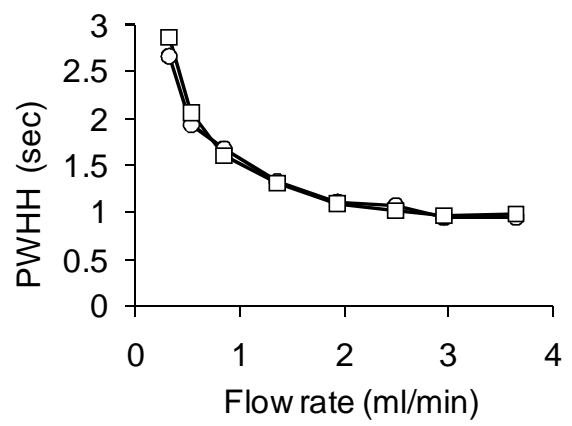

Figure 3: Desorption bandwidth (peak width and half height, PWHH) of TCE from the $\mu F$ as a function of desorption flow rate from 0.3 to $3.6 \mathrm{~mL} / \mathrm{min}$ for TCE alone (squares) and TCE in a mixture with 9 co-contaminants (circles). A higher flow rate leads to narrower injection bands, and there is no influence on PWHH by other VOCs.

Microcolumn fabrication and stationary-phase deposition methods used in this study have been described previously [8]. Rectangular channels, $150 \mu \mathrm{m}$ wide by $240 \mu \mathrm{m}$ deep, were formed in Si by DRIE. A Pyrex cover was anodically bonded to the lower $\mathrm{Si}$ wafer to seal the channels. The 3-m microcolumns have a $3-\mathrm{cm}$ x 3-cm footprint. Each of the 3-m-long columns was coated with
PDMS using a static coating method and crosslinked in situ The calculated PDMS film thickness is $0.15 \mu \mathrm{m}$. Hexamethyldisilazane (HMDS) is used to passivate the channel walls. The microcolumns can be independently temperatureprogrammed from room temperature to $\sim 200{ }^{\circ} \mathrm{C}$ by integrated heaters using a pulse-width-modulation (PWM) method with a proportional-integral-derivative (PID) algorithm

The chemiresistor $(\mathrm{CR})$ array consists of $8 \mathrm{Au} / \mathrm{Cr}$ interdigital electrodes (IDEs) a $4 \times 2$ layout on thermally grown $\mathrm{SiO}_{2}$ over silicon. Each IDE had 24 finger pairs with $5 \mu \mathrm{m}$ width and spacing and finger lengths of $0.5 \mathrm{~mm}$. The array was capped with Macor lid with ports that accepted inlet and outlet capillaries to create a detector cell with an internal volume of $\sim 3 \mu \mathrm{L}$. The device is mounted on a carrier PCB with a cut-out beneath the sensor to permit contact with a thermo-electric cooler beneath the PCB used to thermostat the array at $20{ }^{\circ} \mathrm{C}$. Chemical alteration of the MPN thiolate monolayer gives rise to differential responses for the different sensors; thiols used include n-octanethiol (C8), 1mercapto-6-phenoxyhexane (OPH), methyl 6-mercaptohexanoate (HME), and 4-mercaptodiphenylacetylene (DPA). Two sensors are coated with each MPN for redundancy.

The individual microfabricated components were mounted on discrete carrier PCBs with high temperature epoxy and then mounted in patterned openings cut in the main PCB. Electrical contacts were made to the PCBs via aluminum wire bonding. Two miniature diaphragm pumps (KNF Neuburger, Trenton, NJ) and six micro-solenoid valves (Lee Co., Westbrook, CT) are mounted beneath the PCB and used to direct air flow. All valves were integrated on a stainless steel manifold (not shown). Short sections of deactivated fused silica capillary tubing $(250 \mu \mathrm{m}$ i.d.) were used to fluidically interconnect the components, and MEMS heater sleeves were used at the inlets and outlets of both microcolumns to avoid cold spots.

The system functions are run automatically with user-defined timing and temperature profiles by routines written and compiled in Labview run on a laptop computer equipped with a USBinterfaced multifunction data acquisition card, (USB-6229 National Instruments, Austin, TX).

\section{Sampler, Pre-trap, and $\mu \mathrm{F}$ Performance}

The pre-trap is designed to capture less volatile interfering background vapors with minimal pressure drop, while allowing TCE to pass through unretained. We found that a 50-mg bed of the graphitized carbon Carbopack B $\left(100 \mathrm{~m}^{2} / \mathrm{g}, 212-250 \mu \mathrm{m}\right.$ o.d.) packed in a thin-walled stainless-steel tube (6-mm i.d.) achieves this goal. Using a 20-L sample volume as the maximum sample volume, all compounds with a vapor pressure below 3 torr are completely retained, and TCE is only partially retained from the first $1.5 \mathrm{~L}$ of sample (minimal retention). The sampler is designed to capture TCE along with other VOCs with similar volatility quantitatively for sample volumes up to $20 \mathrm{~L}$. This has been confirmed using a thin-walled stainless-steel tube $(6 \mathrm{~mm}$ i.d.) packed with $100 \mathrm{mg}$ of Carbopack $\mathrm{X}$ in tests of TCE at concentrations ranging from $1-500 \mathrm{ppb}$, with up to 25 other relevant interferences in the sample, both in dry air and in air at $100 \%$ relative humidity. Note that highly volatile interferences are allowed to pass through the system unretained.

Transfer of TCE from the sampler to the $\mu \mathrm{F}$ must be quantitative. Testing has shown that at a flow rate of $30 \mathrm{~mL} / \mathrm{min}$ heating the sampler to $220{ }^{\circ} \mathrm{C}$ results in complete transfer of TCE to the $\mu \mathrm{F}$ in $<3 \mathrm{~min}$. It was also shown that breakthrough of TCE through the $\mu \mathrm{F}$ under these conditions is $<1 \%$. 


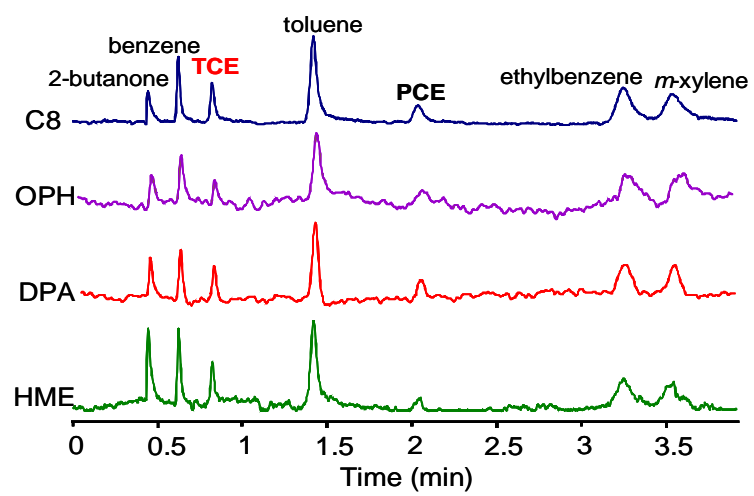

Figure 4. Chromatographic traces from the CR array detector for a preconcentrated test atmosphere of TCE and relevant interferences.

The final step in the preconcentration process is injection of the TCE from the $\mu \mathrm{F}$ to the first microcolumn. For desorption bandwidth determinations, a mass equivalent to $1 \mathrm{ppb}-\mathrm{L}$ was loaded in the $\mu \mathrm{F}$ and the desorption bandwidth was measured as a function of flow rate from 0.3 to $3.6 \mathrm{~mL} / \mathrm{min}$ with the $\mu \mathrm{F}$ heated to $225^{\circ} \mathrm{C}$ within $0.6 \mathrm{sec}$. Tests were performed with TCE alone and also as a mixture with benzene, ethylacetate, heptane, toluene, octane, ethyl benzene, o-xylene, $\alpha$-pinene, and cumene (1 ppb-L each). As shown in Figure 3, the minimum bandwidth of $1 \mathrm{sec}$ is achieved at flow rates $>2 \mathrm{~mL} / \mathrm{min}$ and there is no effect from the presence of VOCs. Since separation efficiency is maximized at flow rates of $0.8-1 \mathrm{~mL} / \mathrm{min}$, this indicates that only a slight tradeoff in separation efficiency is made to operate at a flow rate that gives the sharpest injection band from the $\mu \mathrm{F}$.
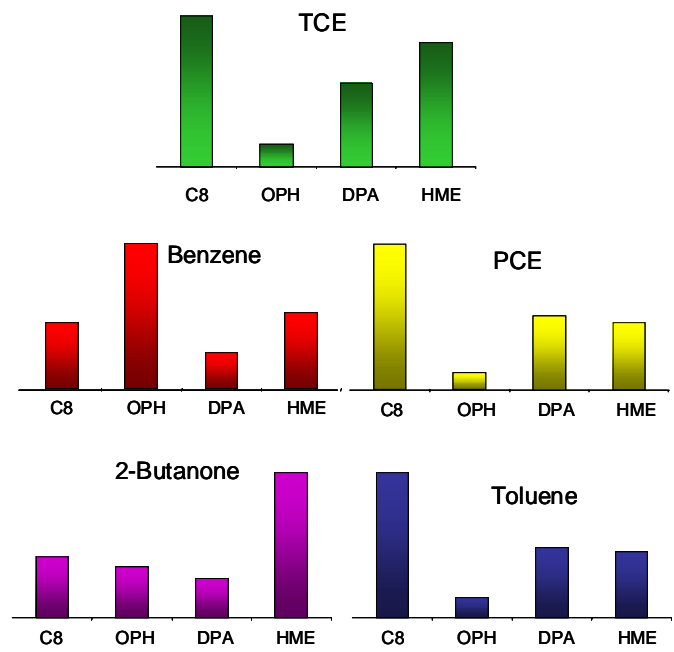

Figure 5: CR sensor array unique responses patterns for TCE, benzene, PCE, 2-butanone and toluene. Response patterns are combined with retention times to recognize and differentiate the VOCs

\section{SPIRON $\mu$ GC TCE determination and calibration}

A 4-L sample of a test-atmosphere containing $4 \mathrm{ppb}$ each of TCE and 6 representative co-contaminants found in homes suffering from VI at our planned field-testing site (2-butanone, benzene, toluene, perchloroethylene (PCE), ethylbenzene, and mxylene, in air) was drawn through the high-volume sampler (pretrap excluded for this test) with an on-board mini-pump. Captured VOCs were thermally desorbed and transferred to the $\mu \mathrm{F}$ and then thermally injected from the $\mu \mathrm{F}\left(200{ }^{\circ} \mathrm{C}\right.$ in $\left.0.4 \mathrm{sec}\right)$ onto the microcolumn ensemble. As shown in Figure 4, TCE was easily separated from the other 6 VOCs and had a retention time of $50 \mathrm{sec}$ with the microcolumns held at room temperature initially and then ramped up to $120^{\circ} \mathrm{C}$ to accelerate elution of the later-eluting interferences.

As shown in Figure 5, unique response patterns are obtained for TCE, benzene, PCE, 2-butanone and toluene from the MPNcoated CR array. Like GC/MS, TCE can be identified by combination chromatographic retention time and unique pattern response.

Figure 6 shows calibration curves for all 4 sensors in the array for TCE with 4-L sample volume. All curves are linear from 2.1 $\mathrm{ppb}$ up to $21 \mathrm{ppb}$ and intersect the origin. As shown in Table 1, for C8 sensor, the calculated LOD for TCE is 0.23 ppt. When the sample volume is increased to $20 \mathrm{~L}, 3$ of the 4 sensors have projected TCE LODs $\leq 70$ ppt.

Table 1. TCE Limits of Detection (LOD, ppb)

\begin{tabular}{|l|c|c|c|c|}
\hline & C8 & OPH & HME & DPA \\
\hline LOD (ppb in 4-L) & 0.23 & 2.9 & 0.32 & 0.35 \\
\hline LOD (ppb in 20-L) & 0.047 & 0.58 & 0.064 & 0.07 \\
\hline
\end{tabular}

\section{Multivariate curve resolution analysis}

Rapid analysis by $\mu \mathrm{GC}$ often results in partial co-elution (overlap) of peaks, which can reduce the ability to identify and quantify the components. An advantage of using a sensor-array detector is that it permits the use of multivariate curve resolution (MCR) to detect hidden peaks in a chromatogram and to resolve overlapped peaks at any degree of chromatographic resolution.

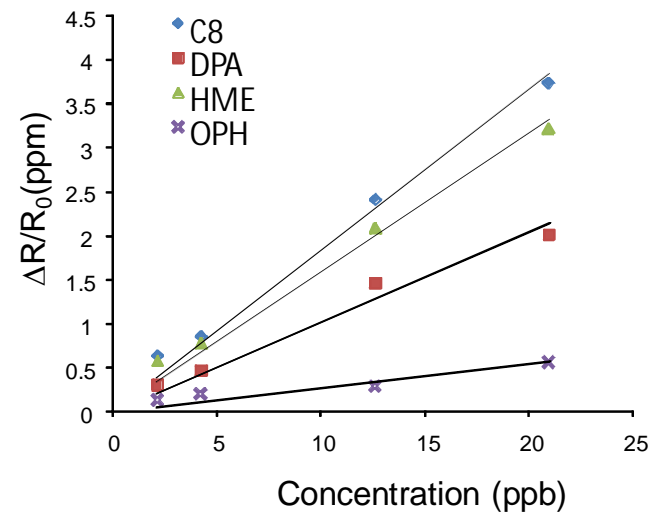

Figure 6. TCE Calibration curves for each of the $4 \mathrm{MPN}$ coated CR sensors in the SPIRON $\mu G C$.

In the previous study [12], we developed an MCR algorithm that combines evolving factor analysis with adaptive least-squares. It was applied successfully to simulated data to illustrate the performance of the technique with a CR array used as a GC detector. We are now applying this technique to experimental data. To illustrate, we chose TCE and heptane as possibly coeluting compounds, since heptane is often found as a cocontaminant in VI-impacted homes and these two VOCs will overlap in analyses performed with the SPIRON $\mu \mathrm{GC}$. Response patterns for heptane and TCE were generated experimentally, and the degree of overlap (chromatographic resolution, $\mathrm{R}$ ) was varied 
by simulation (Matlab) from a value of 0.25 (nearly complete overlap) to 1.0 (nearly completely resolved).
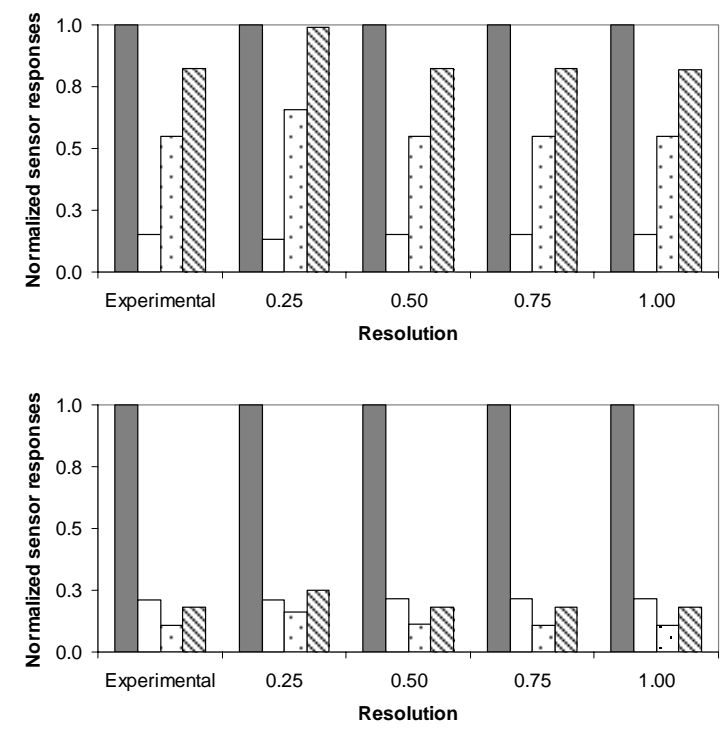

Figure 7. Comparison of response patterns obtained from fully resolved peaks (experimental) of TCE (top) and heptane (bottom) and those recovered from MCR analysis of overlapping peaks of TCE and heptane for different simulated degrees of overlap (resolution). Bars: from left to right: $C 8, O P H, D P A$, and HME.

Figure 7 shows the response pattern for each individual compound along with the recovered response pattern for different degrees of chromatographic resolution ( $\mathrm{R}$ ranges from $0.25-1.0$ ). As shown, the patterns for these co-eluting compounds were recovered with high fidelity (correlation coefficients $\geq 0.98$ ), for all $R$ values. This methodology is being applied to all possible overlapping compounds to guide the separation strategies.

\section{CONCLUSIONS}

First results from a prototype MEMs micro gas chromatograph $(\mu \mathrm{GC})$ adapted for the determination of TCE at low-/sub-ppb concentrations in homes and offices suffering from vapor intrusion have been presented. A front-end pre-trap and high-volume sampler (non-MEMS) have been incorporated to reduced analysis time and facilitate achieving desired LODs. Efficient capture and transfer of TCE samples at lowe concentrations have been confirmed experimentally, along with sharp injection bands form the microfocuser. TCE was separated a subset of anticipated interferences in $\sim 50 \mathrm{sec}$ and response patterns from the CR array, when combined with retention time, permit facile discrimination of TCE from resolved interferences. An MCR technique has been developed to address possible co-eluting interferences in samples with more complex mixtures of interferences. Calibrations are linear from 2-20 ppb. Projected detection limits for a 20-L sample, which can be analyzed in $<30$ minutes, are as low as $50 \mathrm{ppt}$.

\section{ACKNOWLEDGEMENTS}

The authors want to thank Katharine Beach for MEMs device fabrication, Brendan Casey for wire bonding, Robert Gordenker for PCB carrier board design, and David Burris and Jim Reisinger for critical technical guidance. This work was supported by DoD ESTCP ER 200702 through a subcontract with Integrated Science
\& Technology, Inc. and by the National Science Foundation Engineering Research Centers Program (WIMS Center) ERC9986866.

\section{REFERENCES}

[1] M. D. Hsieh, E.T. Zellers, "Limits of recognition for simple vapor mixtures determined with a microsensor array," Anal. Chem., 76, 1885 (2004).

[2] J. C. Jin, P. Kurzawski, A. Hierlemann, E.T. Zellers, "Evaluation of multitransducer arrays for the determination of organic vapor mixtures," Anal. Chem., 80, 227 (2008).

[3] C. J. Lu, W. Steinecker, W. C. Tian, M. Oborny, J. Nichols, M. Agah, J. Potkay, H. Chan, J. Driscoll, R. D. Sacks, K. Wise, S. Pang, E. T. Zellers, "First-generation hybrid MEMS GC," Lab-on-Chip, 5,1123-31 (2005).

[4] E. T. Zellers, S. Reidy, R. A. Veeneman, R. Gordenker, W. H. Steinecker, G. R. Lambertus, H. Kim, J. A. Potkay, M. P. Rowe, Q. Zhong, C. Avery, H. K. L. Chan, R. D. Sacks, K. Najafi, K. D. Wise "An integrated micro-analytical system for complex vapor mixtures," Proc. Transducers '07, Lyon, FR, June 10-14, 2007, pp. 1491-1496.

[5] P.R. Lewis, R.P. Manginell, D.R. Adkins, R.J. Kottenstette, D.R. Wheeler, S.S. Sokolowski, D.E. Trudell, J.E. Bymes, M. Okandan, J.M. Bauer, R.G. Manley, G.C. Frye-Mason, "Recent advancements in the gas-phase $\mu$ ChemLab", IEEE Sensors J., 6, 784 (2006).

[6] S. Zampolli, I. Elmi, F. Mancarella, P. Betti, E. Dalcanale, G. C. Cardinali, M. Severi, "Real-time monitoring of sub-ppb concentrations of aromatic volatiles with a MEMS-enabled miniaturized gas-chromatograph,". Sens. Actuator B-Chem. 141, 322 (2009).

[7] D. N. Cox, W. B. Howard, M. A. Smith, " Heuristic model for predicting the intrusion rate of contaminant vapors into buildings" Environmental science \& technology 25, 1445 (1991).

[8] G. Serrano, S. M. Reidy, E. T. Zellers, "Assessing the reliability of wall-coated microfabricated gas chromatographic separation columns," Sens. Actuator BChem., 141, 217-226 (2009).

[9] S. K. Kim, H. Chang, E .T. Zellers, "Prototype microGC for breath biomarkers of respiratory disease" Proc. Transducers '09, Denver, Colorado, USA, June 21-25, 2009, pp. 128-131.

[10] G. Serrano, H. Chang, E. T. Zellers, A micro gas chromatograph for high-speed determinations of explosive vapors, Proc. Transducers '09, Denver, CO, June 21-25, 2009, pp. 1654-1657.

[11] F. Bohrer, E. Covington, Ç. Kurdak, E. Zellers, "Nanoscale chemiresistor arrays with patterned nanoparticle interface layers for microGC," Proc. Transduc.'09, Denver,CO, June 21-25, 2009, 148-151.

[12] C. Jin and E. T. Zellers, "Chemometric analysis of gas chromatographic peaks measured with a microsensor array: methodology and performance assessment," Sensors and Actuators B-Chemical, 139, 548-556 (2009).

\section{CONTACT}

*Edward T. Zellers, tel: +1-734-936-0766; ezellers@ umich.edu 